\title{
A REINTERPRETATION OF SCHÖNFINKEL'S LOGICAL OPERATORS
}

\author{
BY W. V. QUINE*
}

The method in mathematical logic invented by Schönfinkel $\dagger$ and developed in detail by Curry $\$$ is important in that it completely eliminates the variable from the formal presuppositions of logic and mathematics. Constructed according to Schönfinkel's scheme the primitive language of mathematical logic consists only of a few constants; variables, if wanted as a convenience, are introduced afterward through conventions of shorthand.

Central to Schönfinkel's scheme is the device of construing relations as unitary operators. This is accomplished in the case of a dyadic relation $\phi$ by construing the proposition $\phi x y$ ( $x$ bears the relation $\phi$ to $y$ ) as $(\phi x) y$, that is, as the proposition predicating of $y$ an attribute $\phi x$ which, in its turn, is the result of applying an operator $\phi$ to $x$. (The present recourse to variables is expository only, and foreign to the formal system.) Thus dyadic relations are for Schönfinkel unitary operators yielding attributes, where attributes may, for uniformity, be regarded in turn as unitary operators yielding propositions. In general, any $n$ adic relation is construed in corresponding fashion by taking the proposition $\phi x_{1} x_{2} \cdots x_{n}$ as $\left(\cdots\left(\left(\phi x_{1}\right) x_{2}\right) \cdots\right) x_{n} ; n$-adic relations become unitary operators yielding unitary operators $\cdots$ yielding unitary operators yielding propositions.

My present concern is to point out that Schönfinkel's explanation of relations as unitary operators is gratuitous: inessential to the net interpretation of his formulas, and avoidable by a slight reinterpretation of his notation. The new interpretation is advanced not necessarily as an improvement in an intuitive

* Society of Fellows, Harvard University.

$\dagger$ Mathematische Annalen, vol. 92 (1924), pp. 305-316.

$\ddagger$ American Journal of Mathematics, vol. 51 (1929), pp. 363-384; ibid., vol. 52 (1930), pp. 509-536, 789-834; ibid., vol. 54 (1932), pp. 551-558; Annals of Mathematics, (2), vol. 32 (1931), pp. 154-180; ibid., vol. 34 (1933), pp. 381404 ; ibid., vol. 35 (1934), pp. 849-860; Proceedings of the National Academy of Sciences, vol. 20 (1934), pp. 584-590. 
way, but as a means of correlating the Schönfinkel-Curry scheme with the more familiar one and facilitating translation from one language in to the other.

In addition to the operators, which figure as elements of the Schönfinkel-Curry system, it is apparent from the above that the system involves a binary operation: the operation of application (Curry's term), symbolized by simple juxtaposition. For example, ' $\phi x$ ' indicates the result of applying the operator $\phi$ to $x$. The reinterpretation consists in reconstruing this juxtapositive notation as denoting no longer the operation of application, but rather the operation of deriving what I have elsewhere* called the collective relate, a generalization of the notion $\overleftarrow{R}^{\prime} x$ of Principia Mathematica. Where $\phi$ is an $n$-adic relation, the collective relate $\phi x_{1}$ is the $(n-1)$-adic relation exhibited by objects $x_{2}$ to $x_{n}$ (in that order, understood throughout) if and only if the objects $x_{1}$ to $x_{n}$ exhibit the relation $\phi$. Where $n=2$, $\phi x_{1}$ is thus a "monadic relation" or attribute, that, namely, of being an object to which $x_{1}$ bears the dyadic relation $\phi$. In the degenerate case where $n=1$, the collective relate $\phi x_{1}$ is to be taken as the proposition predicating of $x_{1}$ the attribute $\phi$; this case thus remains as with Schönfinkel.

Thus reinterpreted, the juxtapositive notation ceases to involve the notion of relations as unitary operators and comes rather to express an ordinary operation of relational theory which is independent of any special doctrine as to the nature of relations. The reinterpretation further assimilates the Schönfinkel scheme to usual logistical procedure in that it rids the former of the peculiarity of containing operators as elements. It will now be shown that the reinterpretation has no effect upon the net import of formulas or entire propositional expressions, in other words, that where $\phi$ is $n$-adic the formula ' $\phi x_{1} x_{2} \cdots x_{n}$,' short for ' $\left(\cdots\left(\left(\phi x_{1}\right) x_{2}\right) \cdots\right) x_{n}$,' continues to express the proposition that the objects $x_{1}$ to $x_{n}$ exhibit the relation $\phi$. As reinterpreted $\phi x_{1}$ is the $(n-1)$-adic relation which objects $y_{2}$ to $y_{n}$ exhibit if and only if the objects $x_{1}$ and $y_{2}$ to $y_{n}$ exhibit the $n$-adic relation $\phi$; hence $\left(\phi x_{1}\right) x_{2}$, being the $(n-2)$-adic relation which objects $y_{3}$ to $y_{n}$ exhibit if and only if $x_{2}$ and $y_{3}$ to $y_{n}$ exhibit the $(n-1)$-adic relation $\phi x_{1}$, is

*A System of Logistic, 1934, p. 166. 
exhibited by objects $y_{3}$ to $y_{n}$ if and only if $x_{1}, x_{2}$, and $y_{3}$ to $y_{n}$ exhibit $\phi$. By $n-1$ such cumulative steps of interpretation, we find $\left(\cdots\left(\left(\phi x_{1}\right) x_{2}\right) \cdots\right) x_{n-1}$ to be the "monadic relation" or attribute of being an object $y_{n}$ such that the objects $x_{1}$ to $x_{n-1}$ and $y_{n}$ exhibit $\phi$. The whole proposition applies this attribute to $x_{n}$ and thus tells us that the objects $x_{1}$ to $x_{n}$ exhibit $\phi$.

HARVARD UNIVERSITY

\section{REMARK ON A RECENT PAPER BY HOLLCROFT}

\section{BY A. R. WILLIAMS}

1. Introduction. Among the characteristics of the general web of quadric hypersurfaces in $S_{r}$ described by T. R. Hollcroft in a recent paper* is the number of lines on the jacobian surface of the web, that is, the number of hyperquadrics belonging to the web that have a line of vertices. This and more difficult questions are treated elegantly by associating the hyperquadrics of the web with the planes of a three-space. A direct algebraic treatment of the first mentioned problem may be of interest.

2. Algebraic Formulation of the Problem. The Web of Conics. For a quadric to have a line of vertices it is necessary and sufficient that all the first minors, but not all the second minors, of its discriminant vanish. This is three essential conditions. That is, in the linear system, or web, $\lambda_{1} f_{1}+\lambda_{2} f_{2}+\lambda_{3} f_{3}+\lambda_{4} f_{4}$, where the $f$ 's are linearly independent quadratic forms in any number of variables, a certain number have a line of vertices. While not strictly necessary it will make for clearness to begin with a web of conics. Let the discriminant be

$$
\left|\begin{array}{lll}
a_{11} & a_{12} & a_{13} \\
a_{21} & a_{22} & a_{23} \\
a_{31} & a_{32} & a_{33}
\end{array}\right|,
$$

where the elements are linear homogeneous functions of $\lambda_{1}, \lambda_{2}, \lambda_{3}$, $\lambda_{4}$, and $a_{i j}=a_{j i}$. The three first minors in the first two rows represent quadrics which have a cubic $k$ in common. Any two of them

* This Bulletin, vol. 41 (1935), p. 97. 